\title{
Clinical value of prenatal echocardiographic examination in the diagnosis of fetal cardiac tumors
}

\author{
QIN YU, WENHUA ZENG, AIYUN ZHOU, WAN ZHU and JUAN LIU \\ Department of Ultrasonic Diagnosis, The First Affiliated Hospital of Nanchang University, \\ Nanchang, Jiangxi 330006, P.R. China
}

Received September 4, 2014; Accepted June 2, 2015

DOI: $10.3892 / \mathrm{ol} .2015 .4061$

\begin{abstract}
The aim of the present study was to explore the clinical value of prenatal echocardiographic examination in the diagnosis of fetal cardiac tumors. In total, the cases of 8 fetuses with fetal cardiac tumors, which were identified by prenatal ultrasound examination in The First Affiliated Hospital of Nanchang University between January 2012 and January 2014, were retrospectively analyzed. The size, shape, location, activity and hemodynamic changes of the lesions were described in detail, and the patients were followed up. Out of the 8 identified cases of fetal cardiac tumors, 2 fetuses contained tumors only in the left ventricular cavity and 6 fetuses contained tumors of the left and right ventricular cavities, interventricular septum and apex of the heart. Overall, 5 of the 8 female patients requested termination of the pregnancy and labor was induced. The fetuses were pathologically confirmed to possess rhabdomyoma. In addition, 1 patient was followed-up for 5 weeks, and the tumor in the fetal heart cavity was found to have enlarged and developed in multiple regions when follow-up was performed. The patient then requested termination of the pregnancy and labor was induced. The fetus was pathologically confirmed to possess rhabdomyoma. The remaining 2 patients insisted on continuation of the pregnancy and the fetuses were followed up during gestation and subsequent to birth by echocardiographic examination. Prenatal echocardiography may precisely position and diagnose occupying lesions of the fetal heart, which is of considerable value in clinical decision making and instruction for treatment.
\end{abstract}

Correspondence to: Dr Aiyun Zhou, Deparment of Ultrasonic Diagnosis, The First Affiliated Hospital of Nanchang University, 17 Yongwaizhengjie Street, Nanchang, Jiangxi 330006, P.R. China E-mail: qinyudoc@163.com

Key words: prenatal echocardiographic examination, fetal heart, occupying lesions, rhabdomyoma

\section{Introduction}

The majority of fetal cardiac tumors are primary tumors (1) that usually occur in the heart or pericardial cavities, with rhabdomyoma being the most common tumor type (2). Fetal cardiac tumors are rare, with a global incidence rate of $\sim 0.14 \%$ (2).Notably, rhabdomyoma is frequently associated with tuberous sclerosis (2). Heart tumors are 20-30 times more common than pericardial tumors and $75 \%$ of primary heart tumors are benign; malignant heart tumors are identified less frequently (3). In 1982, DeVore et al used prenatal ultrasonic examination to diagnose fetal cardiac tumors and to evaluate the function of the fetal heart $(4,5)$. Since then, ultrasonic examination has been widely used for fetal screening, allowing for the majority of fetal cardiac tumors to be detected (6). However, successful resection of such tumors is rare, and literature regarding intraoperative monitoring is limited. On ultrasonic examination, rhabdomyoma generally exhibits a uniform high echo, a myocardial echo which is stronger than normal and tuberous sclerosis may also be present. The identification of multiple tumors is common, and such tumors may cause significant hemodynamic disturbances as a result of outflow obstruction or arrhythmias (7). The occurrence of cardiac tumors may cause numerous types of serious blood disorder in fetuses and neonates and may cause fetal mortality. Thus, it is necessary to dynamically monitor the fetal cardiac hemodynamic change (8) and to continuously evaluate the function of the fetal heart by echocardiography $(9,10)$. Accurate prenatal ultrasonic examination of the fetal heart demonstrates important clinical value for the treatment and clinical decision making of fetal cardiac tumors (11).

\section{Materials and methods}

Patients. Between January 2012 and January 2014, 9113 female patients underwent obstetric ultrasound examination at The First Hospital of Nanchang University (Nanchang, Jiangxi, China), and 8 of the fetuses were found to possess occupying lesions of the fetal heart. In total, 2 out of 8 patients were primipara and the other 6 were multipara. The patients were aged between 20 and 35 years old, with a gestational age between 21 and 34 weeks. None of the patients possessed a history of family genetic diseases or 
birth history of fetus malformation. The effect of sib mating was not considered in the present study. The current study was conducted in accordance with the Declaration of Helsinki (12) and with approval from the Ethics Committee of Nanchang University (Nanchang, Jiangxi, China). Written informed consent was obtained from all participants.

Detection of fetal heart. Voluson E8 TruScan (GE Healthcare, Chalfont St Giles, UK) and IU22 Color Doppler Ultrasonic Diagnostic System (Philips, Amsterdam, Netherlands) were used in the present study. A virtual convex probe (GE Healthcare) was used at the frequency of $3.5 \mathrm{MHz}$. The fetal heart model was chosen to observe the four cavities, long axis of the ventriculus sinister, outflow tract of the left and right ventriculus, three vessels view, three vessels and trachea view, short axis of the main artery, aortic arch and ductus arteriosus arch view, in order to determine whether the structure of the heart and blood flow were normal. This was also performed to comprehensively identify the presence of space-occupying masses in the heart cavity, ventricular wall or pericardial cavity at multiple angles. The location, morphology, size, quantity and activity of the masses could then be accurately described. Combined with the spectrum Doppler ultrasonography, the effect of the masses on the left and right outflow tract and atrioventricular and semilunar valves was determined. The induction of fetal cardiac hemodynamic changes was also determined, in addition to the presence of abnormal cardiac function (4).

Detection of the complications. To comprehensively identify whether the fetus possessed complications such as other malformations outside the heart, the ultrasonic probe was switched to the common middle-late pregnancy ultrasound mode. In this mode, it was mainly determined whether there were abnormal echoes or space-occupying masses in the other fetal organs or brain. All imaging data were entered into the ultrasonic workstation at The First Hospital of Nanchang University. Written and informed consent for the use of the prenatal ultrasound examination was obtained from each patient.

\section{Results}

Out of the 8 fetuses identified to possess fetal cardiac tumors, 2 fetuses possessed tumors located within one cardiac ventricle and 6 fetuses possessed tumors located in multiple ventricular cavities or ventricular walls. In total, 6 patients requested termination of the pregnancy and the other 2 patients continued with the pregnancy. Out of the 8 fetuses with cardiac tumors, only 2 demonstrated arrhythmia.

All 8 fetuses possessed a hyperechoic or slightly hyperechoic mass, without evident pedicle, and the masses did not move in large amplitude with cardiac contraction and diastole. No significant hemodynamic disorder or induced obstruction of valves was identified (Table I).

In total, 1 patient (No. 4; Table I) continued with the pregnancy and the fetus was followed up during pregnancy and subsequent to birth by cardiac echocardiographic examination (Fig. 1).

One fetus (No. 2; Table I) was found to possess a 10x6 mm oval high echo, with wide basement membrane but without pedicle, in the left ventricular cavity of the fetal heart at the first ultrasonic examination at the gestational age of

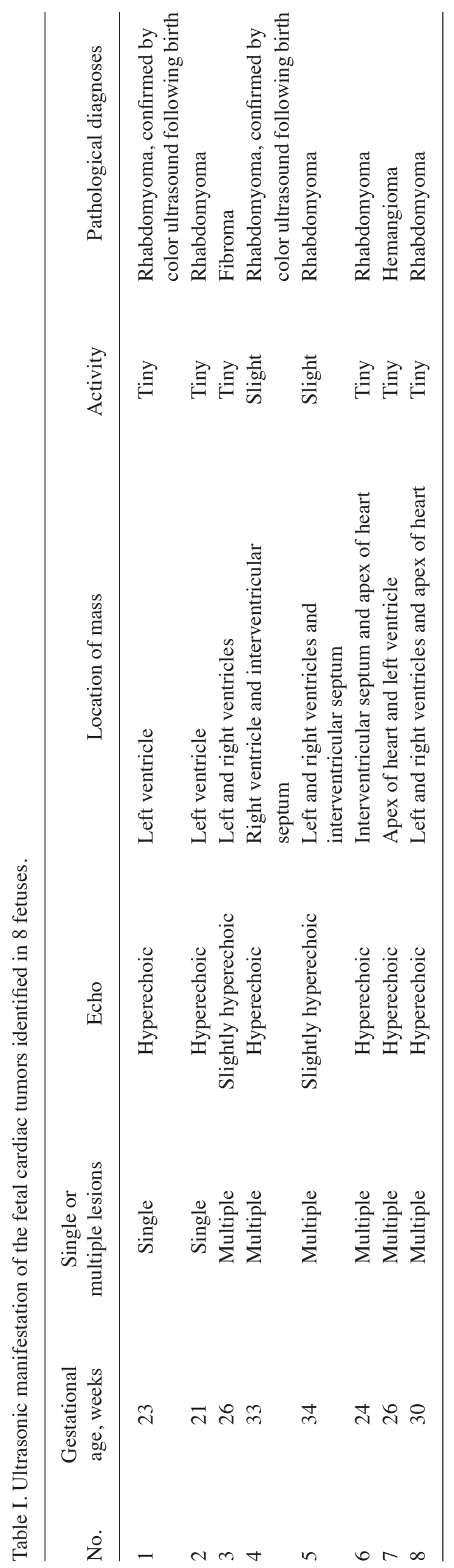



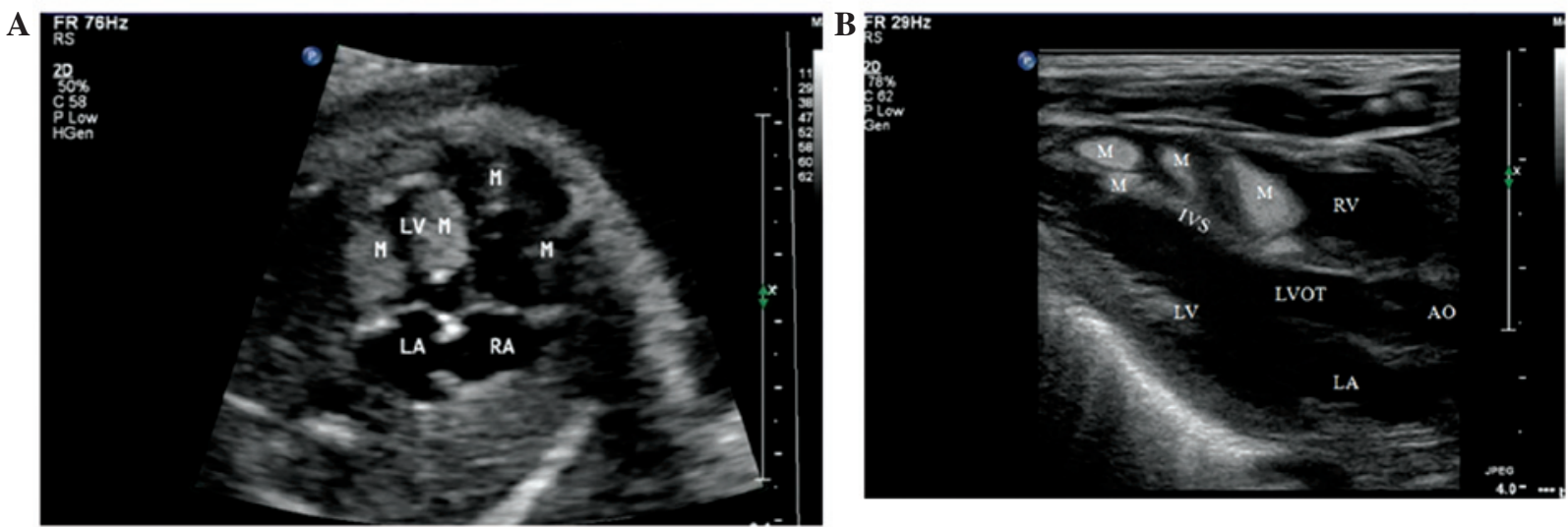

Figure 1. Fetal echocardiography of a pregnant woman at 34 weeks gestation revealing multiple tumors located in the RV and ventricular wall of the fetal heart. The patient chose to continue the pregnancy and during follow-up the cardiac tumors were confirmed by echocardiography. (A) Fetal echocardiography performed on a pregnant woman at 34 weeks gestation, revealing multiple occupying masses located in the ventricular wall and chambers of the fetal heart. (B) Echocardiography performed on the 1-day-old neonate using a superficial probe detected the occupying masses in the RV cavity and interventricular septum. M, masses; LV, left ventricle; LA, left atrium; RV, right ventricle; RA, right atrium; AO, aorta; LVOT, left ventricular outflow tract; IVS, interventricular septum.
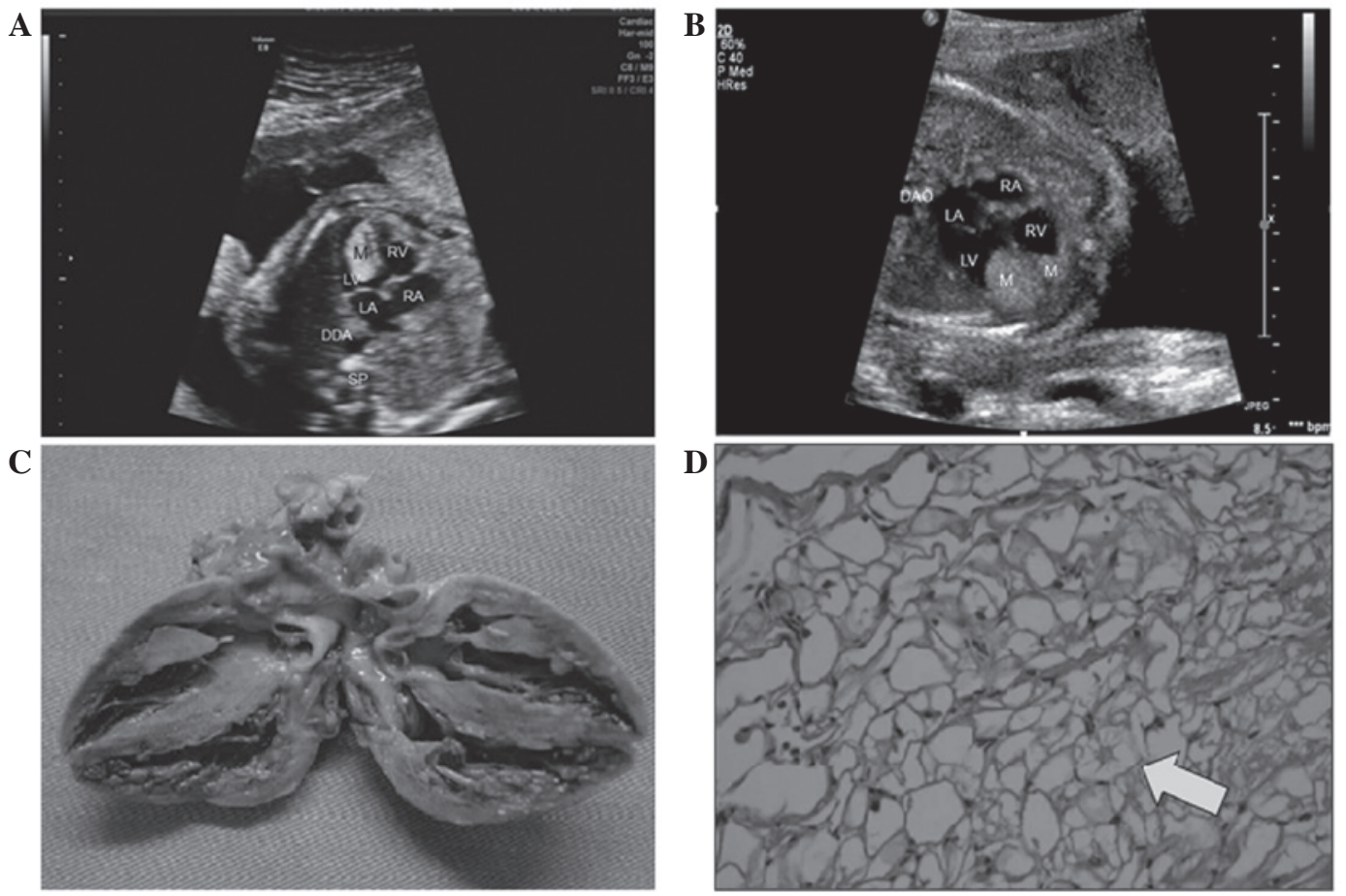

Figure 2. Fetal electrocardiography performed on a pregnant woman at 21 weeks gestation revealed a single tumor located in the LV of the fetal heart. After 5 weeks, the patient was followed up and it was found that the hyperechoic mass in the left ventricular cavity of the fetal heart had increased, and another hyperechoic mass was found in the right ventricular cavity. The patient then chose to terminate the pregnancy. Autopsy examination confirmed that the masses were rhabdomyoma. (A) Fetal electrocardiography performed on a pregnant woman at 21 weeks gestation revealed a single tumor located in the LV of the fetal heart. (B) Fetal electrocardiography performed on a pregnant woman at 26 weeks gestation revealed two masses located in the LV and RV of the fetal heart. (C) Anatomic image of the fetal heart captured subsequent to autopsy. (D) Microscopic histological observation of rhabdomyoma revealing that the cells were vacuolated in unequal sizes and the nuclei were located in the center of the cells, with a small amount of surrounding cytoplasm in a spider-like radial distribution (arrow). Stain, hematoxylin and eosin; magnification, x400. M, masses; LV, left ventricle; RV, right ventricle; LA, left atrium; RA, right atrium; DAO, descending aorta; SP, spinal.

21 weeks (Fig. 2A). This pregnancy was continued and a follow-up was performed. At 26 weeks gestation, a check-up was performed and it was found that the hyperechoic mass in the left ventricular cavity of the fetal heart had increased to $\sim 16 \times 10 \mathrm{~mm}$ and an additional hyperechoic mass $7 \times 6 \mathrm{~mm}$ in size was found in the right ventricular cavity (Fig. 2B). The patient then chose to terminate the pregnancy. Autopsy examination confirmed that the masses were rhabdomyoma (Fig. 2C and D) and there were multiple nodules in each of the fetal kidneys.

\section{Discussion}

Fetal cardiac tumors mostly occur as primary tumors and the incidence rate accounts for $1 / 10,000$ cases of fetal heart 
disease, among which $>90 \%$ are benign (1). Rhabdomyoma remains the most common histological type of fetal cardiac tumor at present, accounting for $60 \%$ of lesions, followed by teratoma, fibroids and hemangioma (13-16).

As detected using echocardiography, fibroma demonstrates equal echo and teratoma demonstrates an uneven echo, usually in the pericardium, while hemangioma demonstrates low echo nodules, with internal tubular echo, or exhibits rich blood flow signals by Doppler ultrasonic examination (11). Rhabdomyoma generally exhibits uniform high echo, stronger than normal myocardial echo, and are usually multiple space-occupying lesions $(17,18)$. Rhabdomyoma may be combined with tuberous sclerosis (19) or damage to other organs, including brain, kidney and liver damage (6). In the present study, the fetal cardiac tumor in 1 fetus demonstrated an increase in size and number when follow-up was performed, while the nodular shadows in the fetal kidneys did not exhibit any evident abnormal echo. The subsequent autopsy examination identified nodules in the kidneys (20). It has been reported that a small number of rhabdomyoma lesions demonstrate a diminishing trend during the years subsequent to birth, and certain lesions disappear naturally (21). Certain studies have even considered fetal cardiac rhabdomyoma to not be a true tumor, but as a hamartoma (22).

When a small and single occupying lesion is identified in the fetal heart, the patient should receive dynamic observation and close follow up (21). However, systemic examination of the nervous system and organs of the fetus should be performed using magnetic resonance imaging (MRI) to identify any damage to other organs (23). In the present study, 2 patients continued with the pregnancy when occupying lesions in the fetal heart were identified. Follow-up was performed for 1 patient from early gestation, subsequent to the identification of a single and small occupying lesion in the fetus, while the other patient was in late gestation and no more abnormality was identified during the follow-up period. Each of the 2 patients underwent MRI but no other evident pathological change was found. Under these circumstances, the fetus may survive. However, it is reported that the recurrence rate is $\leq 50 \%$ (24) and the prognosis is poor. Thus, pregnancy should be terminated when recurrence is identified (25), as a more serious hemodynamic disorder is also likely to occur in the neonatal period and lead to neonatal mortality or neurological symptoms, or other severe complications (26). Therefore, all patients in the present study were constantly followed up prior to the time of writing.

Ultrasonic examination possesses numerous characteristics, such as non-invasiveness, a lack of radiation, repeatability and high diagnostic rate, which indicates that this examination is the best choice for detecting fetal cardiac space-occupying lesions (10). With the constant improvement of ultrasonographic technology and the improved knowledge of diagnostic ultrasonography, early cardiac tumors may be accurately detected (24). However, this should be differentiated with strong echo spots in the ventricular cavity and certain normal heart structures, such as the small papillary muscle (25). Therefore, it should be dynamically and closely followed up in order to avoid misdiagnosis or missed diagnosis (21). During the follow up period, the observations should be compared with the previous examination, to enable the observation of changes in tumor size and the resulting abnormal heart function or blood stream obstruction. Close and dynamic prenatal follow-up may aid in the reduction of the fatality rate and rapidly allow ultrasonic diagnosis to provide additional important novel clinical information for obstetricians and pediatricians. Therefore, prenatal ultrasonic diagnosis of space-occupying lesions in the fetal heart possesses considerable clinical value and is conducive to a healthy birth and good care in developing countries.

\section{References}

1. Silverman N: Primary cardiac tumors. Ann Surg 191: 127-138, 1980.

2. Smythe JF, Dyck JD, Smallhorn JF and Freedom RM: Natural history of cardiac rhabdomyoma in infancy and childhood. Am J Cardiol 66: 1247-1249, 1990.

3. Stellingwerff GC, Hess J and Bogers AJ: Left ventricular rhabdomyoma. A case report. J Cardiovas Surg (Torino) 40: 131-133, 1999.

4. DeVore GR, Hakim S, Kleinman CS and Hobbins JC: The in utero diagnosis of an interventricular septal cardiac rhabdomyoma by means of real-time-directed, M-mode echocardiography. Am J Obstet Gynecol 143: 967-969, 1982.

5. DeVore GR: Assessing fetal cardiac ventricular function. Semin Fetal Neonatal Med 10: 515-541, 2005.

6. Van der Hauwaert LG: Cardiac tumors in infancy and childhood. Br Heart J 33: 125-132, 1971.

7. Björkhem G, Lundström NR and Lingman G: Intracardiac rhabdomyomas in neonates: Report of three cases. Acta Paediatr 81: 712-715, 1992.

8. Hamner LH III, Kaye MF and Weingold AB: Difficulty of fetal monitoring in a fetus with intracardiac tumors. Obstet Gynecol 73: 477-481, 1989.

9. Matsui $\mathrm{M}$ and Gardiner H: Current aspects of fetal cardiovascular function. Fetal Matern Med Rev 19: 61-84, 2008.

10. Van Mieghem T, DeKoninck P, Steenhaut P and Deprest J: Methods for prenatal assessment of fetal cardiac function. Prenat Diagn 29: 1193-1203, 2009.

11. Hornberger LK: Role of quantitative assessment in fetal echocardiography. Ultrasound Obstet Gynecol 35: 4-6, 2010.

12. World Medical Association: WMA Declaration of Helsinki - Ethical principles for medical research involving human subjects. http://www.chcuk.co.uk/pdf/Declaration_of_ Helsinki_2008_Version.pdf. Accessed October 20, 2014.

13. Schmaltz AA and Apitz J: Primary heart tumors in infancy and childhood: Report of four cases and review of literature. Cardiology 67: 12-22, 1981.

14. Jentarra GM, Rice SG, Olfers S, Saffen D and Narayanan V: Evidence for population variation in TSC1 and TSC2 gene expression. BMC Med Genet 12: 29, 2011.

15. McAllister HA Jr: Primary tumors of the heart and pericardium. Pathol Annu 14: 325-355, 1979.

16. Foster ED, Spooner EW, Farina MA, Shaher RM and Alley RD: Cardiac rhabdomyoma in the neonate: Surgical management. Ann Thorac Surg 37: 249-253, 1984.

17. Roach ES, Smith M, Huttenlocher P, Bhat M, Alcorn D and Hawley L: Diagnostic criteria: Tuberous sclerosis complex. Report of the Diagnostic Criteria Committee of the National Tuberous Sclerosis Association. J Clin Neurol 7: 221-224, 1992.

18. Mühler EG, Kienast W, Turniski-Harder V and von Bernuth G: Arrhythmias in infants and children with primary cardiac tumours. Eur Heart J 15: 915-921, 1994.

19. Jóźwiak S,KotulskaK,Kasprzyk-Obara J,Domańska-PakiełaD, Tomyn-Drabik M, Roberts P and Kwiatkowski D: Clinical and genotype studies of cardiac tumors in 154 patients with tuberous sclerosis complex. Pediatrics 118: e1146-e1151, 2006.

20. Chen CP, Su YN, Hung CC, Shih JC and Wang W: Novel mutation in the TSC2 gene associated with prenatally diagnosed cardiac rhabdomyomas and cerebral tuberous sclerosis. J Formos Med Assoc 105: 599-603, 2006.

21. Dal Prato L, Borini A, Coticchio G, Cattoli M and Flamigni C: Half-dose depot triptorelin in pituitary suppression for multiple ovarian stimulation in assisted reproduction technology: A randomized study. Hum Reprod 19: 2200-2205, 2004. 
22. Yim SF, Lok IH, Cheung LP, Briton-Jones CM, Chiu TT and Haines CJ: Dose-finding study for the use of long-acting gongadotrophin-releasing hormone analogues prior to ovarian stimulation for IVF. Hum Reprod 16: 492-494, 2001.

23. Sonigo P, Elmaleh A, Fermont L, Delezoíde AL, Mirlesse V and Brunelle F: Prenatal MRI diagnosis of fetal cerebral tuberous sclerosis. Pediatr Radiol 26: 1-4, 1996.

24. Gresser CD, Shime J, Rakowski H, Smallhorn JF, Hui A and Berg JJ: Fetal cardiac tumor: A prenatal cardiographic marker for tuberous sclerosis. Am J Obstet Gynecol 156: 689-690, 1987.
25. Holley DG, Martin GR, Brenner JI, Fyfe DA, Huhta JC, Kleinman CS, Ritter SB and Silverman NH: Diagnosis and management of fetal tumors. A multicenter experience and review of published reports. J Am Coll Cardiol 26: 516-520, 1995.

26. Bertolini P, Meisner H, Paek SU and Sebening F: Special considerations on primary cardiac tumors in infancy and childhood. Thorac Cardiovasc Surg 38 (Suppl 2): 164-167, 1990. 